\title{
Embedding Topological $n$-Manifolds into Compact Nonstandard Topological $n$-Manifolds with Boundary
}

\author{
Yu-Lin Chou*
}

\begin{abstract}
We show as a main message that there is a simple dimension-preserving way to openly and densely embed every topological manifold into a compact "nonstandard" topological manifold with boundary. This class of "nonstandard" topological manifolds with boundary contains the usual topological manifolds with boundary. In particular, the Alexandroff one-point compactification of every given topological $n$-manifold is a "nonstandard" topological $n$-manifold with boundary.
\end{abstract}

MSC 2020: $57 \mathrm{~N} 35$

Keywords: Alexandroff compactification; embeddings; nonstandard topological manifolds with boundary; topological manifolds

By a manifold we always mean a topological manifold, namely a second-countable Hausdorff space where every point has some neighborhood homeomorphic to some open subset of some (fixed) Euclidean space $\mathbb{R}^{n}$.

By a nonstandard manifold with boundary we always mean a second-countable Hausdorff space where every point has some neighborhood homeomorphic to some subset of some (fixed) upper-half plane $\mathbb{H}_{+}^{n}:=\left\{\left(x_{1}, \ldots, x_{n}\right) \in \mathbb{R}^{n} \mid 0 \leq x_{n}<+\infty\right\}$ such that this subset less the topological boundary $\partial \mathbb{H}_{+}^{n}$ of $\mathbb{H}_{+}^{n}$ is an open set in $\mathbb{R}^{n}$; thus every manifold (with boundary or not) is a nonstandard manifold with boundary. In any given topological space, we will correspondingly refer to a pair of a neighborhood of a given point and a homeomorphism of the neighborhood onto a subset of $\mathbb{H}_{+}^{n}$ of the type specified in the definition of a nonstandard manifold with boundary as a nonstandard chart of the given space about the given point.

The phrase "openly and densely embeddable" always means that an in-context topological embedding has range being an open and dense subset of the in-context codomain.

*Institute of Statistics, National Tsing Hua University, Hsinchu 30013, Taiwan, R.O.C.; Email: y.l.chou@gapp.nthu.edu.tw 
We should like to show with a simple elementary proof that one can openly and densely embed any given $n$-manifold into a compact nonstandard $n$-manifold with boundary:

Theorem 1. Every $n$-manifold is openly and densely embeddable into some compact nonstandard n-manifold with boundary.

Proof. Let $M$ be an $n$-manifold. Since $M$ is in addition locally compact, let $M_{\infty}$ be the Alexandroff (one-point) compactification of $M$ with $\infty$ denoting the additional element and with the only innocuous modification that $\{\infty\}$ is not open in $M_{\infty}$ by considering only compact proper subsets of $M$. Since then $M_{\infty}$ is the only closed set in $M_{\infty}$ that includes $M$, the set $M$ is dense (yet still open) in $M_{\infty}$. This modification is evidently redundant when $M$ is not a compact space.

Choose a nonempty compact proper subset $K$ of $M$ and a precompact coordinate open subset $G$ of $M \backslash K$; and let $\varphi$ be a homeomorphism between $G$ and some open subset of the Euclidean subspace $\mathbb{R}_{++}^{n}:=\left\{\left(x_{1}, \ldots, x_{n}\right) \mid 0<x_{i}<+\infty\right.$ for all $\left.1 \leq i \leq n\right\}$.

If $G_{\infty}:=G \cup\{\infty\}$, let $\varphi_{\infty}: G_{\infty} \rightarrow \mathbb{H}_{+}^{n}$ with $\left.\varphi_{\infty}\right|_{G}:=\varphi$ and $\varphi_{\infty}(\infty):=0$. Then $\varphi_{\infty}$ is a bijection between $G_{\infty}$ and the union of $\{0\}$ and the $\varphi$-image $\varphi^{1)}(G)$ of $G$.

We first claim that $\varphi_{\infty}$ is continuous. To this end, let $A \subset G$. Since a closed proper subset of $M_{\infty}$ is by construction precisely either a compact set in $M$ or the union of $\{\infty\}$ and a closed set in $M$, and since $G$ is precompact, the closure $\operatorname{cl}_{M}(G)$ of $G$ in $M$ is a closed subset of $M_{\infty}$ that includes $A$ (and that does not contain $\infty$ ); and therefore it follows from the definition of closure that

$$
\operatorname{cl}_{G_{\infty}}(A)=\operatorname{cl}_{G}(A)
$$

By this equality, we will then succinctly write $\operatorname{cl}(A)$ for the closure of any subset $A$ of $G$ with respect to either topology. Moreover, the above equality implies that $\infty$ is never an element of the closure of any given subset of $G$.

Fix $A \subset G_{\infty}$. If $\infty \notin A$, then

$$
\varphi_{\infty}^{1)}(\operatorname{cl}(A))=\varphi^{1)}(\operatorname{cl}(A)) \subset \operatorname{cl}_{n}\left(\varphi^{1)}(A)\right)=\operatorname{cl}_{n}\left(\varphi_{\infty}^{1)}(A)\right)
$$

by the continuity of $\varphi$. Here $\operatorname{cl}_{n}(\cdot)$ denotes the closure operation in $\mathbb{H}_{+}^{n}$. If $\infty \in A$, then, since $\{\infty\}$ is closed in $M_{\infty}$, we have

$$
\operatorname{cl}_{G_{\infty}}(A)=\operatorname{cl}(A \backslash\{\infty\}) \cup\{\infty\}
$$

and so

$$
\varphi_{\infty}^{1)}\left(\operatorname{cl}_{G_{\infty}}(A)\right)=\varphi^{1)}(\operatorname{cl}(A \backslash\{\infty\})) \cup\{0\} \subset \operatorname{cl}_{n}\left(\varphi^{1)}(A \backslash\{\infty\})\right) \cup \operatorname{cl}_{n}(\{0\})=\operatorname{cl}_{n}\left(\varphi_{\infty}^{1)}(A)\right)
$$

The continuity of $\varphi_{\infty}$ then follows. 
To show that $\varphi_{\infty}^{-1}$ is continuous, we claim that $\varphi_{\infty}$ is a closed map. But, given any closed set $F$ in $G_{\infty}$, if $\infty \notin F$ then

$$
\varphi_{\infty}^{1)}(F)=\varphi^{1)}(F) \not \ngtr 0
$$

is closed in $\varphi_{\infty}^{1)}\left(G_{\infty}\right)$; whereas if $\infty \in F$, then, since $F \backslash\{\infty\}$ is a closed set in $G$ as previously argued, it follows that

$$
\varphi_{\infty}^{1)}(F)=\varphi^{1)}(F \backslash\{\infty\}) \cup\{0\}
$$

is also closed in $\varphi_{\infty}^{1)}\left(G_{\infty}\right)$.

Now $\left(G_{\infty}, \varphi_{\infty}\right)$ is a nonstandard chart of $M_{\infty}$ about $\infty$. Upon choosing an atlas of $M$ where every coordinate map has range being an open subset of $\mathbb{R}_{++}^{n}$, we have proved that $M_{\infty}$ is a nonstandard $n$-manifold with boundary. Moreover, since $M_{\infty}$ is compact by construction, and since the inclusion map $\left.\operatorname{id}_{M_{\infty}}\right|_{M}: M \rightarrow M_{\infty}$ openly and densely embeds $M$ into $M_{\infty}$, the proof is complete.

Remark. In the proof of Theorem 1, second-countableness evidently plays merely a nominal role; the proof serves every locally compact Hausdorff space having a coordinate open cover (with the evident corresponding verbal changes).

Moreover, the proof of Theorem 1 is evidently more informative, in asserting that every $n$-manifold is an open and dense subspace of a compact nonstandard $n$-manifold with boundary.

For ease of reference, we state the technically evident yet interesting implication of the proof of Theorem 1 in the following

Proposition 1. The Alexandroff one-point compactification of every given n-manifold is a nonstandard n-manifold with boundary. 